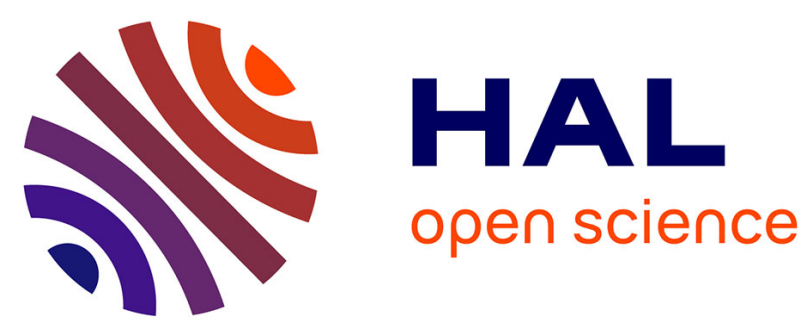

\title{
A fully Automatic and Multi-Structural Segmentation of the Left Ventricle and the Myocardium on Highly Heterogeneous 2D Echocardiographic Data
}

\author{
Sarah Leclerc, Thomas Grenier, Florian Espinoza, Olivier Bernard
}

\section{To cite this version:}

Sarah Leclerc, Thomas Grenier, Florian Espinoza, Olivier Bernard. A fully Automatic and MultiStructural Segmentation of the Left Ventricle and the Myocardium on Highly Heterogeneous 2D Echocardiographic Data. 2017 IEEE International Ultrasonic Symposium (IUS), Sep 2017, Washington, DC, United States. 10.1109/ULTSYM.2017.8092632 . hal-01609235

\section{HAL Id: hal-01609235 https://hal.science/hal-01609235}

Submitted on 11 Feb 2019

HAL is a multi-disciplinary open access archive for the deposit and dissemination of scientific research documents, whether they are published or not. The documents may come from teaching and research institutions in France or abroad, or from public or private research centers.
L'archive ouverte pluridisciplinaire $\mathbf{H A L}$, est destinée au dépôt et à la diffusion de documents scientifiques de niveau recherche, publiés ou non, émanant des établissements d'enseignement et de recherche français ou étrangers, des laboratoires publics ou privés. 


\section{A fully automatic and multi-structural segmentation of the left ventricle and the myocardium on highly heterogeneous 2D echocardiographic data}

\author{
Sarah Leclerc \\ CREATIS, CNRS UMR5220, INSERM U1044 \\ Universite de Lyon, Universite Lyon 1, INSA-LYON \\ Lyon, France \\ sarah.leclerc@creatis.insa-lyon.fr
}

\author{
Thomas Grenier \\ CREATIS, CNRS UMR5220, INSERM U1044 \\ Universite de Lyon, Universite Lyon 1, INSA-LYON \\ Lyon, France \\ thomas.grenier@creatis.insa-lyon.fr
}

\author{
Florian Espinosa \\ Cardiovascular department \\ Centre Hospitalier de Saint-Etienne \\ Saint-Etienne, France \\ florian.espinosa@gmail.com
}

\author{
Olivier Bernard \\ CREATIS, CNRS UMR5220, INSERM U1044 \\ Universite de Lyon, Universite Lyon 1, INSA-LYON \\ Lyon, France \\ olivier.bernard@insa-lyon.fr
}

\begin{abstract}
D echocardiography remains nowadays the main clinical imaging modality in daily practice for assessing the cardiac function. This task requires an accurate segmentation of the left ventricle (LV) and myocardium at end diastole (ED) and systole (ES). Because of intrinsic high variability in image quality in ultrasound data, manual interactions are still needed to obtain a precise delineation of the heart structures. This is both time consuming for specialists and not reproducible. In this study, we investigate a machine learning solution based on the Structured Random Forest algorithm to fully automate the segmentation of the myocardium and LV on heterogeneous clinical data. We compare its performance to the semi-automatic state of the art Active Appearance Model (AAM). The competitive results that were achieved lead us to believe that supervised learning may be the key to automatic heart segmentation.

Index Terms-2D Echocardiography, Structured Random Forests, Machine learning, Multiclass segmentation
\end{abstract}

\section{INTRODUCTION AND BACKGROUND}

Segmentation in echocardiography has always been a challenging research theme due to the bad quality of ultrasound images (low signal to noise ratio, artefacts, poor contrast, high variability...). Since it is fast, cheap and safe, this modality remains the most clinically used. Although 3D images of the heart are now technologically available, cardiologists generally manually do the segmentation of 2D acquisitions to establish their medical diagnostic. A reliable and automatic solution would be a great help in daily examinations. We propose to use a learning algorithm to tackle the issue of automatically

Labex PRIMES (ANR-11-LABX-0063) obtaining a segmentation map out of ultrasound slices of the heart.

\section{A. Related work}

As described in [1], the heart's segmentation has for long been approached with bottom-up algorithms and active contours, which naturally struggle due to bad contrast, fuzzy contours and missing edges [2]. This could be partially solved by the use of shape priors methods [3] (active shape models, registration-based techniques) to produce anatomically plausible shapes [4] [5]. These however often require supervision or indications from the cardiologist. [6] For a few years, database-driven approaches have been more and more applied in medical imaging. This trend born in Computer Vision was followed with a little inertia because of the difficulty to construct medical databases. International challenges such as the 3D cardiac ultrasound left ventricule Cetus challenge in 2014 provide access to data and allow direct and meaningful comparisons of algorithms [7]. Machine learning [8] and deep learning approaches [9] show high potential and are intensively investigated. In particular, decision trees algorithms [10] [11] were successfully applied to echography [12] as well as other modalities [13]. The Structured Random Forests (SRF) was introduced by [14] in computer vision and extended by [15] to edge detection. They were later used on the 3D ultrasound Cetus dataset by [16] and by [17] to compute edge probabilities of the endocardium i.e the LV border. Unlike classical Random Forests [11], the SRF predictions 
are not noisy. Their outputs correspond to full structures with clear transitions, as expected in natural images [14].

\section{B. Contributions}

The LV ejection fraction, alongside myocardium deformations, are primary indicators of heart pathologies. In cardiac ultrasounds, extracting both global and local contexts [18] is essential to localize and identify structures of interest. We believe simultaneously segmentating several structures would be sign of a good use of the contextual information and hence of a reliable algorithm. Our additions to the formalism presented in [16] are the following: first, the modification from an edge detection approach to a segmentation one, where each pixel is given a class representing the structure it belongs to. Second, the incorporation of contextual information in manually-crafted features, which we found deeply improved structures' detection. The resulting method is able to efficiently and simultaneously provide segmentations for both the left ventricle and the myocardium.

\section{Multiclass Segmentation by StRUCtURed RANDOM FORESTS}

A forest is an ensemble of decision trees. Each tree is able to make a prediction of its own and is built to be slightly different from the others. That way, the forest's average prediction corrects the bias that could be brought on by over-fitting the training dataset. Each tree is trained on a random subset of the data and has access to a limited amount of features.

\section{A. Random Forests for classification}

SRF are built in a similar manner as classical Random Forests (RF) [8], that is by recursively constructing nodes which split the training data into two subsets according to their given label $\mathrm{Y}$ and their features $\mathrm{X}$. A test $\mathrm{h}$ at node $\mathrm{i}$ is defined by a function with a binary outcome such as :

$$
h_{i}(X, \tau)=\left[X\left(k_{i}\right)<\tau\right]
$$

Two purer subsets are obtained by optimizing an information gain based cost function at each node to find the most suitable test dividing a set $\mathrm{S}$ into two subsets. We use the Shannon entropy :

$$
H(S)=\sum_{y \in Y} p_{y} \log \left(p_{y}\right)
$$

To avoid over-fitting, random selection of tested features $X_{i}(50 \%)$ and restricted selection of thresholds $\tau$ (only those seen in the node data) are used. Regularizing randomness is also included in the data selection used to train each tree (bagging). A leaf is formed when a stopping criteria is met (maximal depth, minimal information gain or minimal number of data at a node).

\section{B. Structured Outputs}

In $\mathrm{RF}$, a tree learns to map pixels to their corresponding class by successive binary tests on features. SRF differ only in that they do not take pixels as input but image patches. They predict instead of a single label a multiclass segmentation patch. Each data patch is characterized by its segmentation $S$ and its feature vector $X_{p}$. To use an information gain based on entropy as split quality evaluation, we need to assign a label to patches. Similar segmentation patches should be associated to the same label. To do so, we extract the class of pixels chosen at random to summarize each segmentation patch in a $1 \mathrm{D}$ vector. At each node, we apply principal component analysis (PCA) on the node data. A label is then assigned according to the sign of the first components and the medioid patch, closest to mean, is chosen as the leaf's subset's representative.

\section{Contextual Features}

Inspired by [15], we compute gradient-type features for every image. A patch is associated to the concatenation of the features of all its pixels, but also to a few pair-wise differences of features. These are computed between pairs of pixels to take into account spatial evolution of edges across the patch. The feature space includes the intensity at the initial resolution, and the magnitude of gradient and histogram of gradient (HOG) computed at several scales. Scales can provide different contextual information, from local to global. Each scale s of the image is obtained by downsampling the image by a factor $2^{*} \mathrm{~s}$ and applying a smoothing triangular filter. The HOG space is divided into 4, resulting in a total of 5 features maps per scale. All are brought back to the initial resolution by bilinear interpolation.

\section{Testing}

At test time, we compute the new image features, split it into patches and submit them to the decision trees successive binary tests. The prediction for a given patch is the representative segmentation of the leaf that was reached. Several trees are trained as independent classifiers and brought together in an ensemble model. The model's ensemble strategy is to assign to each pixel the class that was most voted for. A pixel receives numerous predictions because each tree will make a prediction for each patch that contains it. This way the forest performs a local segmentation where each pixel benefits from neighboring regions predictions. We observe as in [14] that the averaging process on structured outputs smoothes the overall segmentation map.

\section{E. Pre-processing}

We preprocess the data so that intensities over a given image are normalised as in [6]. Post-processing involves the suppression of supposed false positives by keeping only the biggest predicted structure for each class. 


\section{EXPERIMENT AND COMPARISON}

\section{A. Database}

This study aims at developing an automatic segmentation solution that would suit clinical needs. We gathered in collaboration with the University Hospital of SaintEtienne a still on-growing database of 250 patients cardiac cycles, which were segmented by experts at End Diastoly (ED) and End Systoly (ES). The data represents daily examinations acquired with a GE vivid E9, from sick patients to athletes. It is highly heterogeneous not only due to the heterogeneity in patients but also in size of images, zoom, angle of view... We divide it in the ED set, which contains all images at ED, and the ES set to study the performance of the algorithm on both instants independently.

\section{B. Settings}

For both ED and ES, we use 200 patients as train subjects to build a multiclass segmentation forest and the remaining 50 as test subjects. The parameters optimization of the algorithm comes from a previous study on a different dataset, which acted as a validation set. We build 8 trees with the first 100 train patients and 8 others on the last 100 patients. The resulting 16 trees are all able to predict the segmentation on a new image and have each learnt from 400000 2D patches. We compute features at scales 1, 3 and 5 as previously described to provide local, mid-level and global context. A 2D patch of size 64 is summarized by 64 randomly selected pixels and we use the 3 first PCA components to establish labels. Trees maximum depth is settled at 32 but all trees ended up with a smaller depth. This means the other stopping criteria -the minimum number of patches in a leaf (8) and the minimum information gain (1e-10)- were predominants. At test time, we extract patches with a stride of 2 and associate to each pixel the most voted class from all trees on all patches that contain it.

\section{Evaluation}

For a meaningful comparison, we compute three metrics to account for the segmentation quality and evaluate robustness : the Dice, the Mean Average Distance (MAD) and the Hosdorff Distance (HD). Both distances metrics are computed in $\mathrm{mm}$ and relate to the structures borders : the endocardium for the left ventricule (LV) and the epicardium for the myocardium (Myo). We chose not to take into account the metrics outside of the ultrasound sector where there is no image information.

\section{Active Appearance Model}

We compare our fully automatic solution to semiautomatic state of the art Active Shape Model (ASM) presented in [19]. It requires five initialization points from the user: 2 at the basis, 1 at the apex and 2 at the septum. 4 points are on the endocardium border and the last one initiate the myocardium thickness.
TABLE I

END DIASTOLY

\begin{tabular}{lcccc}
\hline Struct & Algorithm & Dice & HD & MAD \\
\hline \hline \multirow{4}{*}{ LV } & AAM & $0.90 \pm 0.04$ & $\mathbf{7 . 2 4} \pm \mathbf{2 . 7 7}$ & $2.84 \pm 1.20$ \\
& MS-SRF & $\mathbf{0 . 9 2} \pm \mathbf{0 . 0 3}$ & $8.20 \pm 4.77$ & $\mathbf{2 . 1 3} \pm \mathbf{0 . 9 1}$ \\
& MS-SRF-r & $0.92 \pm 0.03$ & $7.41 \pm 3.91$ & $2.04 \pm 0.86$ \\
\multirow{4}{*}{ Myo } & AAM & $\mathbf{0 . 9 2} \pm \mathbf{0 . 0 3}$ & $\mathbf{7 . 5 1} \pm \mathbf{2 . 3 7}$ & $2.91 \pm 1.10$ \\
& MS-SRF & $0.88 \pm 0.08$ & $8.51 \pm 6.9$ & $\mathbf{2 . 4 2} \pm \mathbf{1 . 9 5}$ \\
& MS-SRF-r & $0.90 \pm 0.05$ & $6.73 \pm 3.89$ & $2.01 \pm 0.85$ \\
\hline
\end{tabular}

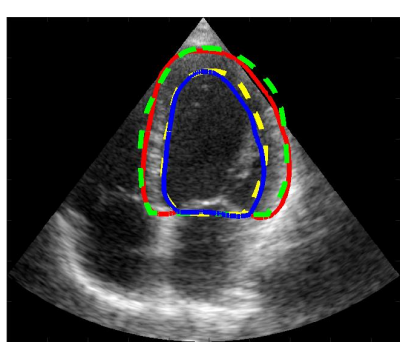

Fig. 1. Segmentation at ED for a test patient with great contrast. Some internal structures in the LV can be seen (papillary muscles, atrioventricular valves) that could lead a classical algorithm astray. The context makes it an easy-to-solve case for the MS-SRF

\section{E. Results}

Tables I and II summarize the results on both datasets and are followed by visual examples of the SRF segmentation performance where ground truth contours are dotted (best seen in color). We use the results on the full test set in our comparison analysis between Multistructural SRF (MS-SRF) and AAM. Though our automatic solution performs generally well, there are 6 cases on which it performs significantly worse. This happens on both ED and ES, which indicates it struggles on specific patients. The most likely reasons for these failures are unusual intensity patterns and contrast as can be observed in figure 3. It is possible that with more training cases, the algorithm could learn to solve them. We also display the statistical results that were obtained without these particular cases (MS-SRF-r), as an indicator to the potential of this method.

We can infer from our experiments that the SRF provide a very interesting fully automatic solution for multistructures cardiac segmentation as its performance is on

TABLE II

END SYSTOLY

\begin{tabular}{lcccc}
\hline Struct & Algorithm & Dice & HD & MAD \\
\hline \hline \multirow{4}{*}{ LV } & AAM & $0.89 \pm 0.06$ & $\mathbf{6 . 9} \pm \mathbf{3 . 0 4}$ & $\mathbf{2 . 2 5} \pm \mathbf{1 . 2 9}$ \\
& MS-SRF & $\mathbf{0 . 9 3} \pm \mathbf{0 . 0 4}$ & $10.23 \pm 5.44$ & $2.88 \pm 1.44$ \\
& MS-SRF-r & $0.93 \pm 0.03$ & $9.04 \pm 4.24$ & $2.57 \pm 1.17$ \\
\hline \multirow{4}{*}{ Myo } & AAM & $\mathbf{0 . 9 3} \pm \mathbf{0 . 0 3}$ & $\mathbf{6 . 6 4} \pm \mathbf{2 . 1 6}$ & $\mathbf{2 . 4 3} \pm \mathbf{0 . 9}$ \\
& MS-SRF & $0.90 \pm 0.08$ & $12.71 \pm 13.14$ & $3.33 \pm 2.53$ \\
& MS-SRF-r & $0.92 \pm 0.04$ & $8.77 \pm 4.50$ & $2.53 \pm 1.16$ \\
\hline
\end{tabular}




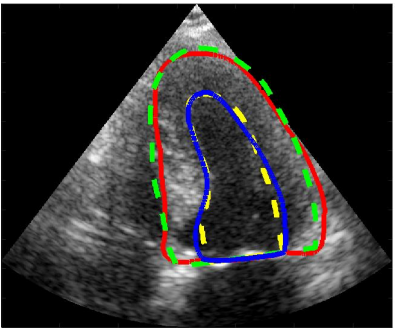

Fig. 2. Segmentation at ES for a test patient with mi-quality contrast. The septum border appears clearly, which helps the overall segmentation.

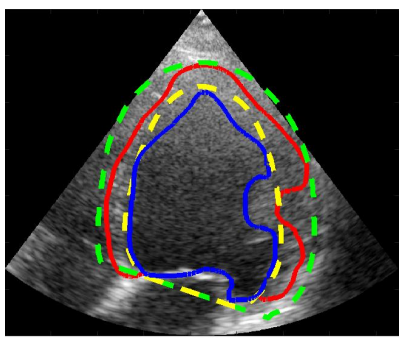

Fig. 3. One of the very difficult case. Very low contrast, unusual intensity patterns and shape configuration are in our opinion responsible.

par with the semi-automatic AAM. It can solve a large variety of configurations as can be seen on the visuals. Thanks to the averaging of local segmentation patches, the predicted structures are coherent, full and closed.

\section{F. Discussion}

The weakness of our algorithm lies in its robustness to very unusual cases. Though there is definitely need for further improvement before considering a clinical application, we deem the results very encouraging. One way to improve robustness would be to learn a lot more data, hoping to learn enough variability to cope with all possible cases. To us, extracting more discriminative features from the image and/or adding shape priors in our formalism are the best leads.

\section{CONCLUSION}

In this paper, we present the framework of our multiclass segmentation algorithm which we apply to 2D echocardiography segmentation. This study is part of a larger one aiming to use machine learning to develop an automatic solution to cardiac segmentation.

\section{ACKNOWLEDGMENT}

This work was supported by the LABEX PRIMES program (ANR-11-LABX-0063) of Universite de Lyon, operated by the French National Research Agency (ANR).

\section{REFERENCES}

[1] G. Carneiro, J.C. Nascimiento, and A. Freitas, "The segmentation of the left ventricle of the heart from ultrasound data using deep learning architecture and derivative-based search methods," IEEE Transactions on Image Processing, vol.21, no .3, pp. 968-982, 2012.
[2] M. Mulet-Parada, J. A. Noble, "2D+T acoustic boundary detection in echocardiography", Med. Image Analysis, vol. 4, pp. 21-30, 2000.

[3] T. Dietenbeck, M. Alessandrini, D. Barbosa, J. D'Hooge, D. Friboulet, $\mathrm{O}$. Bernard, "Detection of the whole myocardium in 2Dechocardiography for multiple orientations using a geometrically constrained level-set," Med. Image Anal., vol. 16, no. 2, pp. 386401, 2012.

[4] N. Paragios, M. P. Jolly, M. Taron, R. Ramaraj, "Active shape models and segmentation of the left ventricle in echocardiography," Proc. Int. Conf. Scale Space Theories PDEs Methods Computer Vision, vol. 3459, pp. 131-142, Apr. 792005.

[5] S. K. Setarehdan, J. J. Soraghan, "Automatic cardiac LV boundary detection and tracking using hybrid fuzzy temporal and fuzzy multiscale edge detection," IEEE Trans. Biomed. Eng., vol. 46, no. 11, pp. 1364-1378, Nov. 1999.

[6] J. G. Bosch, S. C. Mitchell, B. P. F. Lelieveldt, F. Nijland, O. Kamp, M. Sonka, J. H. C. Reiber, "Automatic segmentation of echocardiographic sequences by active appearance motion models," IEEE Trans. Med. Imag., vol. 21, no. 11, pp. 1374-1383, Nov. 2002.

[7] O. Bernard, J.G. Bosch, B. Heyde, M. Alessandrini, D. Barbosa et al., "Standardized Evaluation System for Left Ventricular Segmentation Algorithms in 3D Echocardiography," in IEEE Transactions on Medical Imaging, vol. 35, no. 4, pp. 967-977, April 2016.

[8] A. Criminisi, J. Shotton, and E. Konukoglu, "Decision forests: A unified framework for classification, regression, density estimation,manifold learning and semi-supervised learning," Foundat. Trends Comput.Graph. Vis., vol. 7, no. 2-3, pp. 81-227, 2012.

[9] O. Ronneberger, P. Fischer, T. Brox, "U-Net: Convolutional networks for biomedical image segmentation", Proc. Med. Image Comput. Comput.-Assist. Intervention, pp. 234-241, 2015.

[10] A. Criminisi , J. Shotton, "Decision Forests for Computer Vision and Medical Image Analysis," Springer Publishing Company, Incorporated; 2013. p. 387.

[11] V. Lempitsky, M. Verhoek, J. A. Noble, and A. Blake, "Random forest classification for automatic delineation of myocardium in real-time 3D echocardiography," In International Conference on Functional Imaging and Modeling of the Heart, Springer, Berlin, Heidelberg June 2009. pp. 447-456

[12] S. Ahmadi, K. Boetzel, C. Hennersperger, C. Kroll, F. Milletari, and al, "Robust Segmentation of Various Anatomies in 3D Ultrasound Using Hough Forests and Learned Data Representations," MICCAI. pp 111-118, 2015

[13] A. Criminisi, J. Shotton, S. Bucciarelli, "Decision forests with long-range spatial context for organ localization in CT volumes," MICCAI Workshop on Probabilistic Models for Medical Image Analysis, September 2009

[14] P. Kontschieder, S. R. Bulo, P. Marcello, H. Bischof, Structured Labels in Random Forests for Semantic Labelling and Object Detection, EEE transactions on pattern analysis and machine intelligence. 2014; pp 2104-2116.

[15] P. Dollar, and C. L. Zitnick. "Fast edge detection using structured forests." IEEE transactions on pattern analysis and machine intelligence; 2015. pp.1558-1570.

[16] J. Domingos, R. Stebbing, and J. A. Noble, "Endocardial segmentation using structured random forests in 3D echocardiography," Proc MICCAI Chall Echocardiogr Three-Dimens Ultrasound Segmentation CETUS Boston MIDAS J. 2014; pp. 33-40.

[17] O. Oktay, A. Schuh, M. Rajchl, K. Keraudren, A. Gomez, M. P. Heinrich, et al, "Structured decision forests for multi-modal ultrasound image registration," Proc. Medical Image Computing Computer-Assisted Intervent (MICCAI), pp. 363-371, 2015.

[18] D. Boukerroui, A. Baskurt, J. A. Noble, O. Basset, "Segmentation of ultrasound imagesmultiresolution 2-D and 3-D algorithm based on global and local statistics," Pattern Recognit. Lett., vol. 24, no. 45, pp. 779-790, Feb. 2003

[19] T. F. Cootes, G. J. Edwards, and C. J. Taylor, "Active Appearance Models," IEEE transactions on pattern analysis and machine intelligence. June 2001, pp. 681-685.

[20] D. Barbosa, T. Dietenbeck, B. Heyde, H. Houle, D. Friboulet, J. Dhooge, O. Bernard, "Fast and fully automatic 3-D echocardiographic segmentation using b-spline explicit active surfaces: feasibility study and validation in a clinical setting," Ultrasound Med. Biol., 39 (2013), pp. 89101 